\title{
Stabilizing Sets of PI/PID Controllers for Unstable Second Order Delay System
}

\author{
Rihem Farkh Kaouther Laabidi Mekki Ksouri \\ Laboratory for Analysis, Conception and Control of Systems, LR-11-ES20, Department of Electrical Engineering, National Engineering School \\ of Tunis, Tunis El Manar University, Tunisia
}

\begin{abstract}
In this paper, the problem of stabilizing an unstable second order delay system using classical proportional-integralderivative (PID) controller is considered. An extension of the Hermite-Biehler theorem, which is applicable to quasi-polynomials, is used to seek the set of complete stabilizing proportional-integral/proportional-integral-derivative (PI/PID) parameters. The range of admissible proportional gains is determined in closed form. For each proportional gain, the stabilizing set in the space of the integral and derivative gains is shown to be a triangle.
\end{abstract}

Keywords: Hermite-Biehler theorem, stability, PI/PID controllers, second order unstable delay system, stability region.

\section{Introduction}

Time delay systems are often encountered in various engineering systems such as electrical and communication network, chemical process, turbojet engine, nuclear reactor and hydraulic system. Delay is frequently a source of instability, oscillation and poor performance in many dynamic systems. Furthermore, delay makes system analysis and control design much more complex ${ }^{[1,2]}$. Many processes in industrial and chemical practice are open-loop unstable processes that are known to be difficult to control, such as in the case of polymerization reactors, bioreactors, continuous stirred tank and reactors, exothermic stirred reactors with back mixing, batch reactors, pump with liquid storage tank and combined feed/effluent heat exchanger with adiabatic exothermic reactor, etc., which are inherently openloop unstable by design. Especially, unstable delay processes make control system design a complex task, which has attracted increased attention in the process control community ${ }^{[3]}$. Recently, many academic researches have been devoted to developing proportional-integral -derivative (PID) control strategies for unstable delay processes. Today, proportional-integral (PI) and PID controller types are the most widely used control strategies. It is estimated that $98 \%$ of control loops in the pulp and paper industries are controlled by PI controllers ${ }^{[4]}$ and that in process control applications, more than $95 \%$ of the controllers are of PID type $^{[5]}$. Since the minimal requirement for PID controller is to guarantee the system stability, it is desirable to know the complete set of stabilizing PID parameters before tuning and design. Surveys have reported that poor tuning, configuration errors, traditional and empirical techniques such as Ziegler Nichols or Cohen-Coon tuning methods, are found in the industrial application of $\mathrm{PID}^{[6]}$. A great deal of academic and industrial effort has focused on improving PID control in the areas of tuning rules to decrease the rising gap between engineering practice and control theory. Based on the gain and phase margins criterion, De Paor and $\mathrm{O}^{\prime}$ Malley ${ }^{[7]}$ proposed PID controllers tuning method

Manuscript received January 13, 2013; revised July 23, 2013 for the first order delay unstable plant first order delay plant (FODUP). Quinn and Sanathanan ${ }^{[8]}$ investigated a method for the design of controllers for unstable delayed processes based on model matching in the frequency domain. Shafie and Shenton ${ }^{[9]}$ discussed a graphical technique based on the D-partition method for PID controller tuning for stable and unstable processes. Padma Sree et al. ${ }^{[10]}$ presented PI/PID controllers design for first order unstable delay system by extracting the coefficients of the numerator and denominator of the closed-loop transfer function. Huang and $\operatorname{Lin}^{[11]}$ developed optimum PID tuning for unstable first and second order delay plant (FODUP and SODUP) based on the minimization of the integral of absolute error (IAE) criterion and using the least-squares method. Poulin and Pomerleau $^{[12]}$ studied a graphical tuning method of PI and PID controllers for integrating processes and unstable processes (FODUP and SODUP) which is based on the analysis of the open-loop frequency response of the process on the Nichols chart. In [13], the authors applied D-partition method to characterize the stability domain in the space of controller parameters. The stability boundary is reduced to a transcendental equation, and the whole stability domain is drawn in two-dimensional plane by sweeping the remaining parameters. However, this result only provides sufficient condition regarding the size of the time delay for stabilization of first-order unstable processes. Using Nyquist criterion, Xiang et al. ${ }^{[14]}$ solved the stabilization problem of second-order unstable delay process by PID controller. An enhanced two-degrees-of-freedom control strategy for second-order unstable process with time-delay is established in [15]. Shamsuzoha introduced an enhanced PID controller for unstable first ${ }^{[16]}$ and second order delay plant ${ }^{[17]}$. Chen et al. ${ }^{[16]}$ investigated the calculation of set point weighting PID parameter for unstable first-order time-delay systems. In [18], the internal model controller equivalent PID tuning rules are synthesized for a low order unstable delay systems in [18]. In [19], the authors presented an adaptive iterative learning control scheme for a class of strict-feedback nonlinear time-delay systems with unknown nonlinearly parameterised and time-varying disturbed functions of known 
periods. Chen and $\mathrm{Li}^{[20]}$ developed an observer-based adaptive iterative learning control scheme for a class of nonlinear systems with unknown time-varying parameters and unknown time-varying delays. In [21], the delay-dependent robust stabilization and an $H_{\infty}$ control for uncertain stochastic Takagi-Sugeno fuzzy systems with discrete interval and distributed time-varying delays are discussed. Kumar and Mittal $^{[22]}$ proposed a parallel fuzzy proportional plus fuzzy integral plus fuzzy derivative $(\mathrm{FP}+\mathrm{FI}+\mathrm{FD})$ controller for some complex processes, such as first and second-order processes with delay, inverse response process with and without delay and higher order processes.

Recent studies have made use of the generalization of Hermite-Biehler theorem to compute the set of all stabilizing PID controllers for a given plant ${ }^{[12]}$. Since almost all plants encountered in process control contain time-delays, computing the complete set of PID controllers that stabilize time delay system is of considerable importance. An analytical approach is used in [23-25] to study the characterization of the stability region of delayed systems controlled via PI/PID. Indeed, by using the Hermite-Biehler theorem applicable to the quasi-polynomials [25-27], a characterization of all values of the PI/PID stabilization gains for stable and unstable first order delay system is addressed. However, these results are not applicable to the second order delay system. In $[28,29]$, the stabilizing problem of PI/PID controller for second order delay system is analyzed and then used to obtain all PI and PID gains that stabilize a first and a second order delay interval systems ${ }^{[30,31]}$.

In this paper, we employed a version of the HermiteBiehler theorem applicable to quasi-polynomials to investigate the complete set of stabilizing PI/PID parameters for unstable second order process with time delay. However, all these results mentioned above are centered upon the issues of controller design, synthesis and parameter tuning, which do not deal with the determination of the complete set of stabilizing PID controllers based on stability conditions on unstable time delay systems by PID controllers as shown in our paper. We can also improve our approach by searching optimal PI or PID controller inside the stability region for a given performance criteria (integral of square error (ISE), integral of absolute error (IAE), time multiplied by absolute error (ITAE), time multiplied by square error (ITSE), maximum overshoot, rise time and settling time) by using genetic algorithm with regard to the complexity of the optimization problem as indicated in [28].

\section{Preliminary results for analyzing time delay system}

Several problems in process control engineering are related to the presence of delays. These delays intervene in dynamic models whose characteristic equations are of the following form ${ }^{[23-25]}$ :

$$
\begin{aligned}
\delta(s)= & d(s)+\mathrm{e}^{-L_{1} s} n_{1}(s)+ \\
& \mathrm{e}^{-L_{2} s} n_{2}(s)+\cdots+\mathrm{e}^{-L_{m} s} n_{m}(s)
\end{aligned}
$$

where $d(s)$ and $n_{i}(s)$ are polynomials with real coefficients and $L_{i}$ represent time delays. These characteristic equations are recognized as quasi-polynomials. Under the fol- lowing assumptions

$$
\begin{array}{ll}
\left(A_{1}\right) \quad \operatorname{deg}(d(s))=n \text { and } \operatorname{deg}\left(n_{i}(s)\right)<n \\
& \text { for } i=1,2, \cdots, m \\
\left(A_{2}\right) \quad 0<L_{1}<L_{2}<\cdots<L_{m}
\end{array}
$$

one can consider the quasi-polynomials $\delta^{*}(s)$ described by

$$
\begin{aligned}
\delta^{*}(s)= & \mathrm{e}^{s L_{m}} \delta(s)= \\
& \mathrm{e}^{s L_{m}} d(s)+\mathrm{e}^{s\left(L_{m}-L_{1}\right)} n_{1}(s)+ \\
& \mathrm{e}^{s\left(L_{m}-L_{2}\right)} n_{2}(s)+\cdots+n_{m}(s) .
\end{aligned}
$$

The zeros of $\delta(s)$ are identical to those of $\delta^{*}(s)$ since $\mathrm{e}^{s L_{m}}$ does not have any finite zeros in the complex plane. However, the quasi-polynomial $\delta^{*}(s)$ has a principal term since the coefficient of the term containing the highest powers of $s$ and $\mathrm{e}^{s}$ is nonzero. If $\delta^{*}(s)$ does not have a principal term, then it has infinite roots with positive real parts ${ }^{[23-25]}$.

The stability of the system with characteristic equation (1) is equivalent to the condition that all the zeros of $\delta^{*}(s)$ must be in the open left half of the complex plane. Hence $\delta^{*}(s)$ is Hurwitz or is stable. The following theorem gives a necessary and sufficient condition for the stability of $\delta^{*}(s)$.

Theorem $\mathbf{1}^{[23-25]}$. Let $\delta^{*}(s)$ be given by (3); so

$$
\delta^{*}(\mathrm{j} \omega)=\delta_{r}(\omega)+\mathrm{j} \delta_{i}(\omega)
$$

where $\delta_{r}(\omega)$ and $\delta_{i}(\omega)$ represent the real and imaginary parts of $\delta^{*}(\mathrm{j} \omega)$, respectively. Under conditions $A_{1}$ and $A_{2}$, $\delta^{*}(s)$ is stable if and only if:

1) $\delta_{r}(\omega)$ and $\delta_{i}(\omega)$ have only simple, real roots and these interlace;

2) $\delta_{i}^{\prime}\left(\omega_{0}\right) \delta_{r}\left(\omega_{0}\right)-\delta_{i}\left(\omega_{0}\right) \delta_{r}^{\prime}\left(\omega_{0}\right)>0$ for some $\omega_{0}$ in $[-\infty,+\infty]$.

where $\delta_{i}^{\prime}(\omega)$ and $\delta_{r}^{\prime}(\omega)$ denote the first derivative with respect to $\omega$ of $\delta_{i}(\omega)$ and $\delta_{r}(\omega)$, respectively.

A crucial stage in the application of the preceding theorem is to make sure that $\delta_{r}(\omega)$ and $\delta_{i}(\omega)$ have only real roots. Such a property can be checked while using the following theorem.

Theorem $2^{[23-25]}$. Let $M$ and $N$ designate the highest powers of $s$ and $\mathrm{e}^{s}$ which appear in $\delta^{*}(s)$. Let $\eta$ be an appropriate constant such that the coefficient of terms of highest degree in $\delta_{r}(\omega)$ and $\delta_{i}(\omega)$ do not vanish at $\omega=\eta$. Then, a necessary and sufficient condition that $\delta_{r}(\omega)$ and $\delta_{i}(\omega)$ have only real roots is that in each of the intervals $-2 l \pi+\eta<\omega<2 l \pi+\eta, l=l_{0}, l_{0}+1, l_{0}+2 \cdots, \delta_{r}(\omega)$ or $\delta_{i}(\omega)$ have exactly $4 l N+M$ real roots for a sufficiently large integer $l_{0}$.

\section{PI control for unstable second order delay system}

A second order system with time delay can be mathematically expressed by a transfer function having the following form

$$
G(s)=\frac{K}{s^{2}+a_{1} s+a_{0}} \mathrm{e}^{-L s}
$$

where $K$ is the static gain of the plant, $L$ is the time delay, and $a_{0}$ and $a_{1}$ are the plant parameters. The PI controller 
is described by the following transfer function

$$
C(s)=K_{p}+\frac{K_{i}}{s} .
$$

According to [25], the closed loop characteristic equation of the system is stable at $L=0$, then it will be stable for all $L>0$, whereas if it is unstable for $L=0$, then it will be unstable for all $L>0$. So, a minimal requirement for any control design is that the delay-free closed-loop system be stable.

The characteristic equation of the closed-loop delay-freesystem $(L=0)$ is given by

$$
\begin{aligned}
\delta(s)= & K\left(K_{i}+K_{p} s\right)+\left(s^{2}+a_{1} s+a_{0}\right) s= \\
& s^{3}+a_{1} s^{2}+\left(a_{0}+K K_{p}\right) s+K K_{i} .
\end{aligned}
$$

$\delta(s)$ is a third-order polynomial. The closed-loop stability is equivalent to all its coefficients are non zero and have the same sign, we have

$$
a_{1}>0, \quad a_{0}+K K_{p}>0, \quad K K_{i}>0 .
$$

For $a_{0}>0$ and $a_{1}>0$, we obtain an open-loop stable plant. And for $a_{0}<0$ and $a_{1}>0$, we have an openloop unstable delay plant. In the following, we present the synthesis of PI controller in the case of an unstable second order delay system where $K>0, L>0, a_{0}<0$ and $a_{1}>0$.

We state Theorem 3 determining the range of $K_{p}$ and $K_{i}$ for unstable second order delay system controlled by PI controller.

Theorem 3. Under the assumptions of $K>0, L>0$, $a_{0}<0$ and $a_{1}>0$, the $K_{p}$ values, for which there is a solution for the stabilization problem of the PI controller of unstable second order delay system, verify:

$$
-\frac{a_{0}}{K}<K_{p}<\frac{1}{K}\left(a_{1} \frac{\alpha}{L} \sin (\alpha)-\cos (\alpha)\left(a_{0}-\frac{\alpha^{2}}{L^{2}}\right)\right)
$$

where $\alpha$ is the solution of the equation: $\tan (\alpha)=$ $\frac{\alpha\left(2+a_{1} L\right)}{\left(\alpha^{2}-a_{1} L-a_{0} L^{2}\right)}$ in the interval $[0, \pi]$.

For $K_{p}$ values outside this range, there are no stabilizing PI controllers. The range of $K_{i}$ value is given by

$$
0<K_{i}<\min _{j=1,3,5, \ldots}\left\{a_{j}\right\}
$$

where $a_{j}=a\left(z_{j}\right)=\frac{z_{j}}{K L}\left[\sin \left(z_{j}\right)\left(a_{0}-\frac{z_{j}^{2}}{L^{2}}\right)+a_{1} \frac{z_{j}}{L} \cos \left(z_{j}\right)\right]$ and $z_{j}, j=1,2,3, \cdots$, are the roots (arranged in ascending order of magnitude) of $\delta_{i}(z)=\frac{z}{L}\left(K K_{p}+\cos (z)\left(a_{0}-\frac{z 2}{L^{2}}\right)-\right.$ $\left.a_{1} \frac{z}{L} \sin (z)\right)$.

Proof. The characteristic equation of the closed-loop system is given by

$$
\delta(s)=K\left(K_{i}+K_{p} s\right) \mathrm{e}^{-L s}+\left(s^{2}+a_{1} s+a_{0}\right) s
$$

we deduce the quasi-polynomial $\delta^{*}(s)$ :

$$
\delta^{*}(s)=\mathrm{e}^{L s} \delta(s)=K\left(K_{i}+K_{p} s\right)+s\left(s^{2}+a_{1} s+a_{0}\right) \mathrm{e}^{L s}
$$

by replacing $s$ by j $\omega$, we get

$$
\delta^{*}(\mathrm{j} \omega)=\delta_{r}(\omega)+\mathrm{j} \delta_{i}(\omega)
$$

where

$$
\left\{\begin{array}{l}
\delta_{r}(\omega)=K K_{i}+\left(\omega^{3}-a_{0} \omega\right) \sin (L \omega)-a_{1} \omega^{2} \cos (L \omega) \\
\delta_{i}(\omega)=\omega\left[K K_{p}+\left(a_{0}-\omega^{2}\right) \cos (L \omega)-a_{1} \omega \sin (L \omega)\right]
\end{array}\right.
$$

Clearly, the parameter $K_{i}$ only affects the real part of $\delta^{*}(\mathrm{j} \omega)$ whereas the parameter $K_{p}$ affects the imaginary part.

Let $z=L \omega$, we get:

$$
\left\{\begin{array}{l}
\delta_{r}(z)=K K_{i}+\sin (z)\left(\frac{z^{3}}{L^{3}}-a_{0} \frac{z}{L}\right)-a_{1} \frac{z^{2}}{L^{2}} \cos (z) \\
\delta_{i}(z)=\frac{z}{L}\left(K K_{p}+\cos (z)\left(a_{0}-\frac{z 2}{L^{2}}\right)-a_{1} \frac{z}{L} \sin (z)\right) .
\end{array}\right.
$$

The application of the second condition of Theorem 1 led us to

$$
E\left(z_{0}\right)=\delta_{i}^{\prime}\left(z_{0}\right) \delta_{r}\left(z_{0}\right)-\delta_{i}\left(z_{0}\right) \delta_{r}^{\prime}\left(z_{0}\right)>0
$$

from (11), we have

$$
\begin{aligned}
\delta_{i}^{\prime}(z)= & \frac{K K_{p}}{L}-\sin (z)\left(a_{0}+\frac{2 a_{1} z}{L^{2}}-\frac{z^{3}}{L^{3}}\right)+ \\
& \cos (z)\left(\frac{a_{0}}{L}-\frac{3 z^{2}}{L^{3}}-a_{1} \frac{z^{2}}{L^{2}}\right)
\end{aligned}
$$

for $z_{0}=0$ (a value that annul $\left.\delta_{i}(z)\right)$, we get

$$
E\left(z_{0}\right)=\delta_{i}^{\prime}\left(z_{0}\right) \delta_{r}\left(z_{0}\right)=\left(\frac{K K_{p}+a_{0}}{L}\right) K K_{i}>0
$$

which implies $K_{p}>-\frac{a_{0}}{K}$ since $K>0$ and $K_{i}>0$.

We consider the verification of the interlacing condition of $\delta_{r}(z)$ and $\delta_{i}(z)$ roots. For such purpose, we are going to determine the roots from the imaginary part, which is translated by the following relation:

$$
\begin{aligned}
\delta_{i}(z)=0 & \Rightarrow\left\{\begin{array}{l}
z=0 \\
\text { or } \\
K K_{p}+\cos (z)\left(a_{0}-\frac{z^{2}}{L^{2}}\right)-a_{1} \frac{z}{L} \sin (z)=0
\end{array}\right. \\
& \Rightarrow\left\{\begin{array}{l}
z=0 \\
\text { or } \\
K K_{p}+\cos (z)\left(a_{0}-\frac{z^{2}}{L^{2}}\right)=a_{1} \frac{z}{L} \sin (z)
\end{array}\right. \\
& \Rightarrow\left\{\begin{array}{l}
z=0 \\
\text { or } \\
f(z)=g(z)
\end{array}\right.
\end{aligned}
$$

where

$$
\left\{\begin{array}{l}
f(z)=K K_{p}+\cos (z)\left(a_{0}-\frac{z^{2}}{L^{2}}\right) \\
g(z)=a_{1} \frac{z}{L} \sin (z)
\end{array}\right.
$$

We notice $z_{0}=0$ is a trivial root of the imaginary part. The others are difficult to solve analytically. However, this can be completed graphically. Two cases are presented. In each case, the positive real roots of $\delta_{i}(z)$ will be denoted by $z_{j}, j=1,2,3, \cdots$, arranged in increasing order of magnitude.

Case 1. $-\frac{a_{0}}{K}<K_{p}<K_{u}$. 
In this case, we graph the curves of $f(z)$ and $g(z)$ which are presented in Fig. 1.

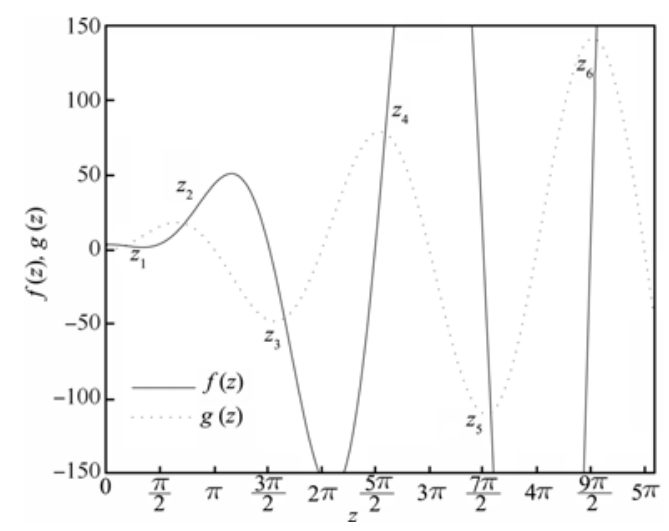

Fig. 1 Representation of the curves of $f(z)$ and $g(z)$ (Case: $\left.-\frac{a_{0}}{K}<K_{p}<K_{u}\right)$

$K_{u}$ is defined in second case. We notice that for $-\frac{a_{0}}{K}<$ $K_{p}<K_{u}$, the curve of $f(z)$ intersects the curve of $g(z)$ twice in the interval $[0, \pi]$. Also we can see the following properties:

$$
\left\{\begin{array} { c } 
{ z _ { 1 } \in [ 0 , \frac { \pi } { 2 } ] } \\
{ z _ { 3 } \in [ \frac { 3 \pi } { 2 } , 2 \pi ] } \\
{ z _ { 5 } \in [ \frac { 7 \pi } { 2 } , 4 \pi ] } \\
{ \vdots }
\end{array} \quad \text { and } \left\{\begin{array}{c}
z_{2} \in\left[\frac{\pi}{2}, \pi\right] \\
z_{4} \in\left[\frac{5 \pi}{2}, 3 \pi\right] \\
z_{6} \in\left[\frac{9 \pi}{2}, 5 \pi\right] \\
\vdots
\end{array}\right.\right.
$$

i.e., $z_{j}$ verifies:

$$
\left\{\begin{array}{l}
z_{1} \in\left[0, \frac{\pi}{2}\right] \\
\text { and } \\
z_{j} \in\left[(2 j-3) \frac{\pi}{2},(j-1) \pi\right] \text { for } j \geqslant 2
\end{array}\right.
$$

and we remark: $z_{j}>z=L \sqrt{a_{0}}, j=1,2,3,5,7, \cdots$.

Case 2. $K_{p} \geqslant K_{u}$.

Fig. 2 represents the case when $K_{p}=K_{u}$, and $K_{u}$ is the maximal value of $K_{p}$, so the plot of $f(z)$ intersects the plot of $g(z)$ twice in the interval $[0, \pi]$ (i.e., $f(z)$ is tangent to $g(z))$.

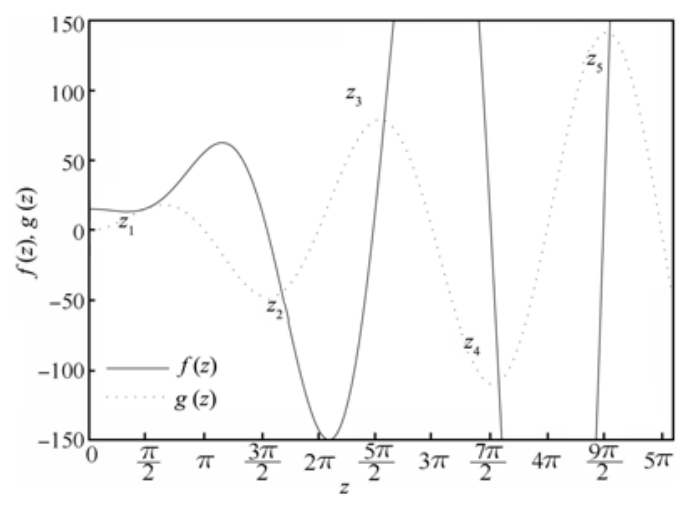

Fig. 2 Representation of the curves of $f(z)$ and $g(z)$ (Case: $\left.K_{p}=K_{u}\right)$

The plot in Fig. 3 corresponds to the case when $K_{p}>K_{u}$ and the plot of $f(z)$ does not intersect $g(z)$ twice in the interval $[0, \pi]$.

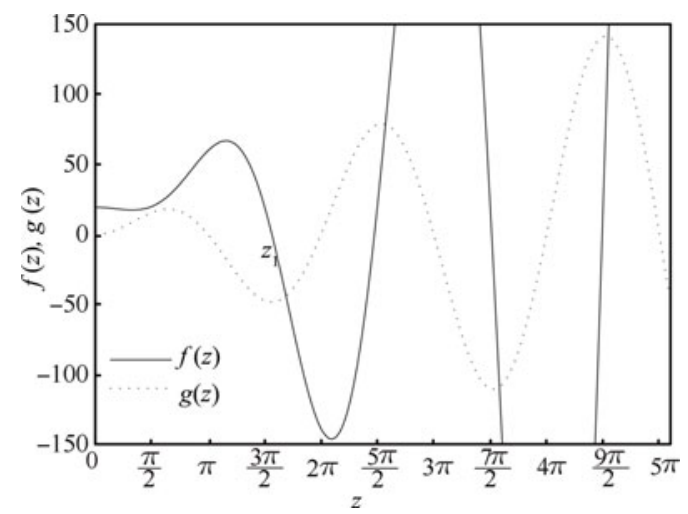

Fig. 3 Representation of the curves of $f(z)$ and $g(z)$ (Case: $\left.K_{p}>K_{u}\right)$

Theorem 2 is used to verify that $\delta_{i}(z)$ possesses only simple roots. By replacing Ls by $s_{1}$ in (8), we rewrite $\delta^{*}(s)$ as

$$
\begin{aligned}
\delta^{*}(s)= & \mathrm{e}^{L s} \delta(s)=\mathrm{e}^{s_{1}} \delta\left(s_{1}\right)= \\
& \mathrm{e}^{s_{1}}\left(\left(\frac{s_{1}}{L}\right)^{3}+a_{1}\left(\frac{s_{1}}{L}\right)^{2}+a_{0} \frac{s_{1}}{L}\right)+K\left(K_{p} \frac{s_{1}}{L}+K_{i}\right) .
\end{aligned}
$$

For this new quasi-polynomial, we see that $M=3$ and $N=1$, where $M$ and $N$ designate the highest powers of $s_{1}$ and $\mathrm{e}^{s_{1}}$ which appear in $\delta^{*}(s)$. We choose $\eta=\frac{\pi}{4}$ that satisfies the condition given by Theorem 2 as $\delta_{r}(\eta) \neq 0$ and $\delta_{i}(\eta) \neq 0$. According to Fig. 1, we notice that for $-\frac{a_{0}}{K}<K_{p}<K_{u}, \delta_{i}(z)$ possesses four roots in the interval $\left[0,2 \pi-\frac{\pi}{4}\right]=\left[0, \frac{7 \pi}{4}\right]$ including the root at origin. As $\delta_{i}(z)$ is odd function, so it possesses seven roots in $\left[-2 \pi+\frac{\pi}{4}, 2 \pi-\frac{\pi}{4}\right]=\left[-\frac{7 \pi}{4}, \frac{7 \pi}{4}\right]$. Hence, we can affirm that $\delta_{i}(z)$ has $4 N+M=7$ roots in $\left[-2 \pi+\frac{\pi}{4}, 2 \pi+\frac{\pi}{4}\right]=$ $\left[-\frac{7 \pi}{4}, \frac{9 \pi}{4}\right]$. At the end, according to Theorem $2, \delta_{i}(z)$ has only real roots for every $K_{p}$ in $\left[-\frac{a_{0}}{K}, K_{u}\right]$. For $K_{p} \geqslant K_{u}$ corresponding Figs. 3 and 4 , the roots of $\delta_{i}(z)$ are not real. We determine the superior bound of $K_{p}$, according to the definition of $K_{u}$, if $K_{p}=K_{u}$, then the curves of $f(z)$ and $g(z)$ are tangent at the point $\alpha$, which is expressed by

$$
\begin{aligned}
& \left\{\begin{array}{l}
K K_{u}+\cos (\alpha)\left(a_{0}-\frac{\alpha^{2}}{L^{2}}\right)=a_{1} \frac{\alpha}{L} \sin (\alpha) \\
\text { and } \\
\frac{\mathrm{d}}{\mathrm{d} z}\left[K K_{u}+\cos (z)\left(a_{0}-\frac{z^{2}}{L^{2}}\right)\right]_{z=\alpha}=\frac{\mathrm{d}}{\mathrm{d} z}\left[a_{1} \frac{z}{L} \sin (z)\right]_{z=\alpha} \\
\Rightarrow-2 \alpha \cos (\alpha)\left(1+a_{1} L\right)+\sin (\alpha)\left(\alpha^{2}-a_{0} L^{2}-a_{1} L\right)=0
\end{array}\right. \\
& \Rightarrow \tan (\alpha)=\frac{\alpha\left(2+a_{1} L\right)}{\left(\alpha^{2}-a_{0} L^{2}-a_{1} L\right)} .
\end{aligned}
$$

Once $\alpha$ is determined, the parameter $K_{u}$ is expressed by

$$
K_{u}=\frac{1}{K}\left(a_{1} \frac{\alpha}{L} \sin (\alpha)-\cos (\alpha)\left(a_{0}-\frac{\alpha^{2}}{L^{2}}\right)\right) .
$$

After the determination of the roots of the imaginary part $\delta_{i}(z)$, we move to the evaluation of these roots by the real 
$\operatorname{part} \delta_{r}(z)$

$$
\begin{aligned}
\delta_{r}(z)= & K K_{i}+\sin (z)\left(\frac{z^{3}}{L^{3}}-a_{0} \frac{z}{L}\right)-a_{1} \frac{z^{2}}{L^{2}} \cos (z)= \\
& K\left[K_{i}-a(z)\right]
\end{aligned}
$$

where $a(z)=\frac{z}{K L}\left[\sin (z)\left(a_{0}-\frac{z^{2}}{L^{2}}\right)+a_{1} \frac{z}{L} \cos (z)\right]$.

Let us denote the roots of $\delta_{i}(z)$ by $z_{j}, j=1,2,3, \cdots$. For $z_{0}$, we have

$$
\delta_{r}\left(z_{0}\right)=K\left(K_{i}-a(0)\right)=K K_{i}>0
$$

for $z_{j} \neq z_{0}$, where $j=1,2,3, \cdots$, we get

$$
\delta_{r}\left(z_{j}\right)=K\left(K_{i}-a\left(z_{j}\right)\right)=K\left(K_{i}-a_{j}\right)
$$

where $a\left(z_{j}\right)=a_{j}$.

Interlacing of the roots of $\delta_{r}(z)$ and $\delta_{i}(z)$ is equivalent to $\delta_{r}\left(z_{0}\right)>0\left(\right.$ since $\left.K_{i}>0\right), \delta_{r}\left(z_{1}\right)<0, \delta_{r}\left(z_{2}\right)>0, \cdots$.

We can use the interlacing property and the fact that $\delta_{i}(z)$ has only real roots to reach that $\delta_{r}(z)$ possesses real roots, too. From the previous equations, we get the following inequalities.

$$
\left\{\begin{array} { c } 
{ \delta _ { r } ( z _ { 0 } ) > 0 } \\
{ \delta _ { r } ( z _ { 1 } ) < 0 } \\
{ \delta _ { r } ( z _ { 2 } ) > 0 } \\
{ \delta _ { r } ( z _ { 3 } ) < 0 } \\
{ \delta _ { r } ( z _ { 4 } ) > 0 } \\
{ \vdots }
\end{array} \Rightarrow \left\{\begin{array}{c}
K_{i}>0 \\
K_{i}<a_{1} \\
K_{i}>a_{2} \\
K_{i}<a_{3} \\
K_{i}>a_{4} \\
\vdots
\end{array} .\right.\right.
$$

From these inequalities, it is clear that the $a_{j}$ odd bounds must be strictly positive. However, $a_{j}$ even bounds are negative in order to find a feasible range of $K_{i}$, from which we have:

$$
0<K_{i}<\min _{j=1,3,5 \ldots}\left\{a_{j}\right\}
$$

In the following, we are interested to prove that the odd $a_{j}^{\prime}$ s are strictly positive and that the even $a_{j}{ }^{\prime}$ s are negative in order to affirm (19).

The change of $a\left(z_{j}\right)$ can be found with a graphical approach. Given a stabilizing $K_{p}$ value inside the admissible range by using Theorem 3 , the curve of $\delta_{i}(z)$ is plotted to obtain their roots denoted by $z_{j}, j=1,2,3 \cdots$, the curve of $a\left(z_{j}\right)$ is also plotted in order to understand its behavior (Fig. 4). We note that for every $K_{p}$ in $\left[-\frac{a_{0}}{K}, K_{u}\right]$, the parameters $a\left(z_{j}\right)$ verify the following conditions:

$$
\left\{\begin{array}{l}
a\left(z_{j}\right)>0, \text { for } j=1,3,5,7, \cdots \\
a\left(z_{j}\right)<0, \text { for } j=2,4,6,8, \cdots .
\end{array}\right.
$$

Algorithm 1. For determining PI parameters for unstable second order delay system:

1) Choose $K_{p}$ in the interval suggested by Theorem 3;

2) Find the roots $z_{j}$ of $\delta_{i}(z)$;

3) Compute the parameter $a_{j}$ associated with the $z_{j}$ previously founded;

4) Determine the lower and the upper bounds for $K_{i}$ as

$$
0<K_{i}<\min _{j=1,3,5 \ldots}\left\{a_{j}\right\} .
$$

5) Go to Step 1).

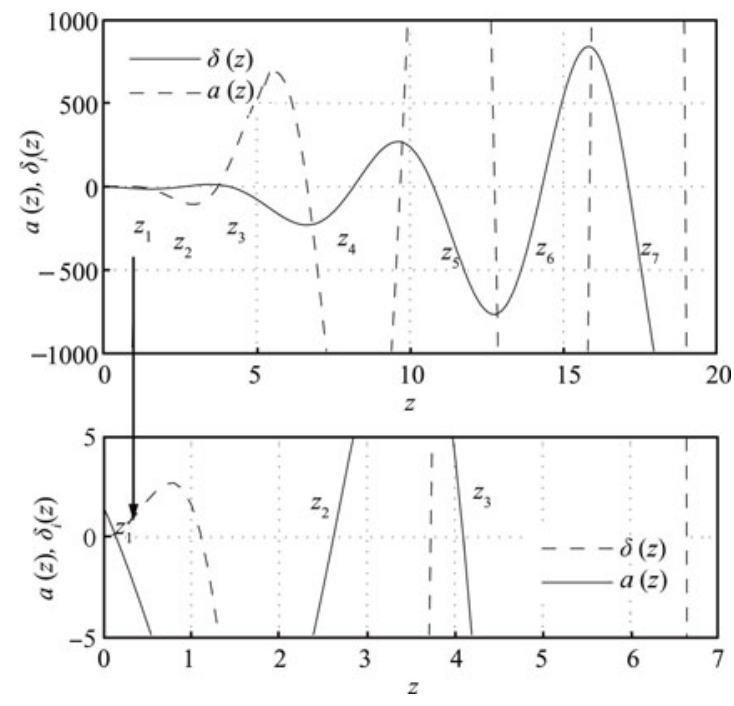

Fig. 4 Plots of $\delta_{i}(z)$ and $a(z)$

Example 1. We consider an unstable second order delay system described by the following transfer function

$$
G(s)=\frac{2 \mathrm{e}^{-0.5 s}}{-0.5+5 s+s^{2}} .
$$

In order to determine $K_{p}$ values, we look for $\alpha$ in interval $[0, \pi]$ satisfying $\tan (\alpha)=\frac{4.5 \alpha}{\alpha^{2}-2.625} \Rightarrow \alpha=1.5617 . K_{p}$ range is given by $0.25<K_{p}<7.85$. We remark

$$
\left\{\begin{array}{l}
0.25<K_{p}<6.2 \quad \Rightarrow \quad K_{i}>0 \\
6.2<K_{p}<7.85 \quad \Rightarrow \quad K_{i}<0 .
\end{array}\right.
$$

In consequence, we choose the final range of $K_{p}$ as $0.25<$ $K_{p}<6.2$.

The controller stability region, obtained in $\left(K_{p}, K_{i}\right)$ plane, is presented in Fig. 5 .

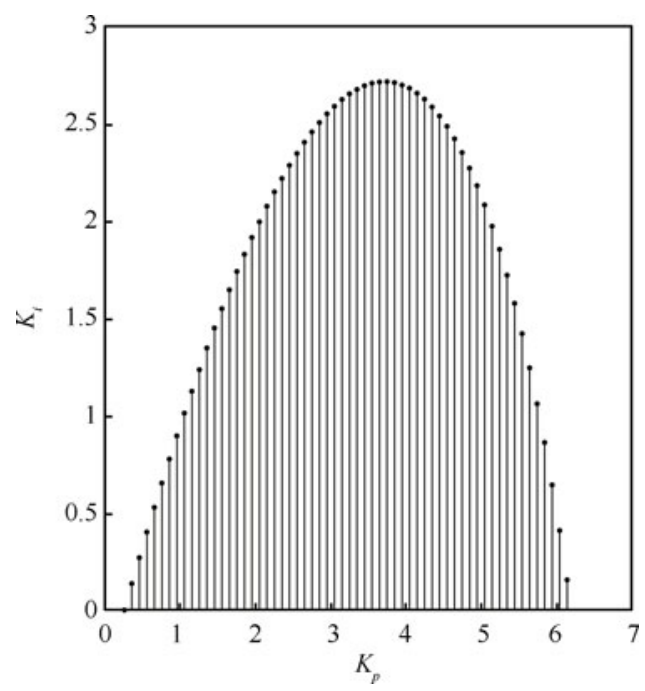

Fig. 5 Controller stability domain in $\left(K_{p}, K_{i}\right)$ for unstable second order delay plant

The step response of the closed-loop system with some PI controllers is shown in Fig. 6. 


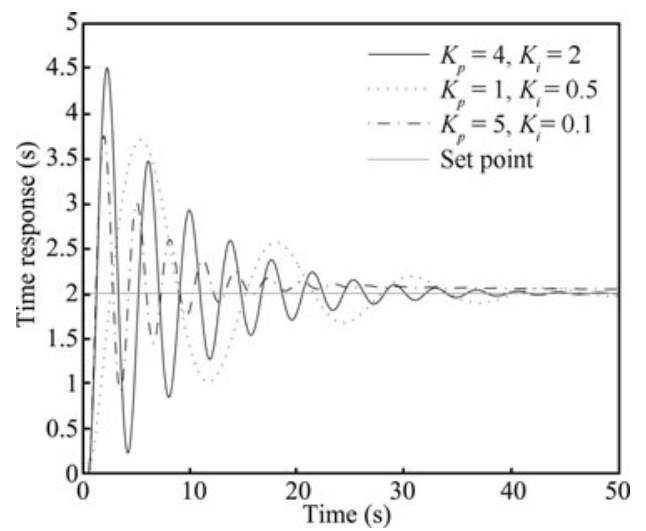

Fig. 6 Time response of the closed-loop system for Example 1

From Fig. 6, we see that the closed-loop system is stable and the output $y(t)$ tracks the step input signal.

\section{PID control for unstable second or- der delay system}

The PID controller is described by the following transfer function:

$$
C(s)=K_{p}+\frac{K_{i}}{s}+K_{d} s
$$

The characteristic equation of the closed-loop delay-freesystem $(L=0)$ is given by

$$
\begin{aligned}
\delta(s)= & K\left(K_{i}+K_{p} s+K_{d} s^{2}\right)+\left(s^{2}+a_{1} s+a_{0}\right) s= \\
& s^{3}+\left(a_{1}+K K_{d}\right) s^{2}+\left(a_{0}+K K_{p}\right) s+K K_{i} .
\end{aligned}
$$

Since this is a third-order polynomial, closed-loop stability is equivalent to all its coefficients are non zero and have the same sign, we have

$$
a_{1}+K K_{d}>0, a_{0}+K K_{p}>0, \quad K K_{i}>0 .
$$

We assume that static gain $K$ of the plant is positive, we obtain the following condition for closed-loop stability of delay-free system

$$
K_{p}>-\frac{a_{0}}{K}, \quad K_{i}>0, \quad K_{d}>-\frac{a_{1}}{K} .
$$

For $a_{0}>0$ and $a_{1}>0$, we obtain an open-loop stable plant. And for $a_{0}<0$ or/and $a_{1}<0$, we have an open-loop unstable delay plant.

In the following, we present the synthesis of PID controller for the case of an unstable second order delay system where $K>0, L>0, a_{0}<0$ or/and $a_{1}<0$.

Theorem 4. Under the above assumptions of $K>0$, $L>0, a_{0}<0$ or/and $a_{1}<0$, the range of $K_{p}$ values for which a solution exists to the PID stabilization problem of an open-loop unstable plant with transfer function $G(s)$ is given by

$$
-\frac{a_{0}}{K}<K_{p}<\frac{1}{K}\left(a_{1} \frac{\alpha}{L} \sin (\alpha)-\cos (\alpha)\left(a_{0}-\frac{\alpha^{2}}{L^{2}}\right)\right)
$$

where $\alpha$ is the solution of the equation $\tan (\alpha)=$ $\frac{\alpha\left(2+a_{1} L\right)}{\left(\alpha^{2}-a_{1} L-a_{0} L^{2}\right)}$ in $[0, \pi]$.
For $K_{p}$ values outside this range, there are no stabilizing PID controllers. The complete stabilizing region given by the cross-section of the stabilizing region in the $\left(K_{i}, K_{d}\right)$ space is the triangle $\Delta$ (Fig. 7 ).

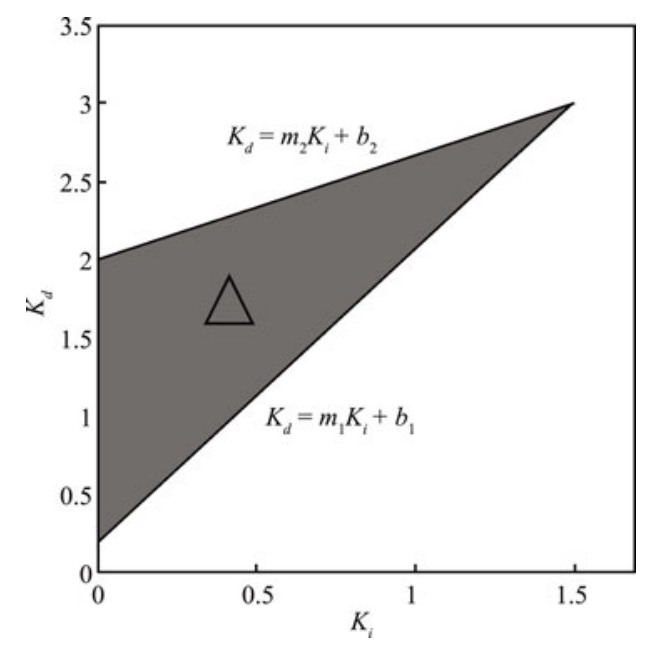

Fig. 7 The stabilizing region of $\left(K_{i}, K_{d}\right)$

The parameters $b_{j}, m_{j}, j=1,2$, necessary for determining the boundaries can be determined using

$$
\left\{\begin{array}{l}
m_{j}=m\left(z_{j}\right)=\frac{L^{2}}{z^{2}} \\
b_{j}=b\left(z_{j}\right)=\frac{L}{K z_{j}}\left[-a_{1} \frac{z_{j}}{L} \cos \left(z_{j}\right)+\sin \left(z_{j}\right)\left(\frac{z_{j}^{2}}{L^{2}}-a_{0}\right)\right]
\end{array}\right.
$$

where $z_{j}, j=1,2$, are the positive-real roots of $\delta_{i}(z)$ arranged in ascending order of magnitude where $\delta_{i}(z)$ is expressed by

$$
\delta_{i}(z)=\frac{z}{L}\left(K K_{p}+\cos (z)\left(a_{0}-\frac{z 2}{L^{2}}\right)-a_{1} \frac{z}{L} \sin (z)\right) .
$$

Proof. The characteristic equation of the closed-loop system is given by

$$
\delta(s)=K\left(K_{i}+K_{p} s+K_{d} s^{2}\right) \mathrm{e}^{-L s}+\left(s^{2}+a_{1} s+a_{0}\right) s .
$$

The quasi-polynomial $\delta^{*}(s)$ is given by

$$
\begin{aligned}
\delta^{*}(s)= & \mathrm{e}^{L s} \delta(s)= \\
& K\left(K_{i}+K_{p} s+K_{d} s^{2}\right)+s\left(s^{2}+a_{1} s+a_{0}\right) \mathrm{e}^{L s} .
\end{aligned}
$$

By replacing $s$ by j $\omega$, we get

$$
\delta^{*}(\mathrm{j} \omega)=\delta_{r}(\omega)+\mathrm{j} \delta_{i}(\omega)
$$

with

$$
\left\{\begin{aligned}
\delta_{r}(\omega)= & K K_{i}-K K_{d} \omega+\left(\omega^{3}-a_{0} \omega\right) \sin (L \omega)- \\
& a_{1} \omega^{2} \cos (L \omega) \\
\delta_{i}(\omega)= & \omega\left[K K_{p}+\left(a_{0}-\omega^{2}\right) \cos (L \omega)-a_{1} \omega \sin (L \omega)\right] .
\end{aligned}\right.
$$

Clearly, the parameters $K_{i}$ and $K_{d}$ only affect the real part of $\delta^{*}(\mathrm{j} \omega)$, whereas the parameter $K_{p}$ affects the imaginary 
part. Letting $z=L \omega$, we get

$$
\left\{\begin{aligned}
\delta_{r}(z)= & K K_{i}-K K_{d} \frac{z^{2}}{L^{2}}+\sin (z)\left(\frac{z^{3}}{L^{3}}-a_{0} \frac{z}{L}\right)- \\
& a_{1} \frac{z^{2}}{L^{2}} \cos (z) \\
\delta_{i}(z)= & \frac{z}{L}\left(K K_{p}+\cos (z)\left(a_{0}-\frac{z 2}{L^{2}}\right)-a_{1} \frac{z}{L} \sin (z)\right) .
\end{aligned}\right.
$$

We notice that $\delta_{i}(z)$ is the same in (10) and (24). Consequently, we obtain the same range of $K_{p}$ values, which is given by Theorem 3 that stabilize an unstable second order delay system.

After determination of the roots of the imaginary part $\delta_{i}(z)$, we evaluate of these roots by the real part $\delta_{r}(z)$.

$$
\begin{aligned}
\delta_{r}(z)= & K K_{i}-K K_{d} \frac{z^{2}}{L^{2}}+\sin (z)\left(\frac{z^{3}}{L^{3}}-\right. \\
& \left.a_{0} \frac{z}{L}\right)-a_{1} \frac{z^{2}}{L^{2}} \cos (z)= \\
& K \frac{z^{2}}{L^{2}}\left\{-K_{d}+K_{i} \frac{L^{2}}{z^{2}}+\frac{L}{K z}\left[-a_{1} \frac{z}{L} \cos (z)+\right.\right. \\
& \left.\left.\sin (z)\left(\frac{z^{2}}{L^{2}}-a_{0}\right)\right]\right\}= \\
& K \frac{z^{2}}{L^{2}}\left[-K_{d}+m(z) K_{i}+b(z)\right]
\end{aligned}
$$

where

$$
\left\{\begin{array}{l}
m(z)=\frac{L^{2}}{z^{2}} \\
b(z)=\frac{L}{K z}\left[-a_{1} \frac{z}{L} \cos (z)+\sin (z)\left(\frac{z^{2}}{L^{2}}-a_{0}\right)\right] .
\end{array}\right.
$$

From condition 1 of Theorem 1 , the roots of $\delta_{r}(z)$ and $\delta_{i}(z)$ have to interlace for $\delta^{*}(s)$ to be stable. We evaluate $\delta_{r}(z)$ at the roots of the imaginary part $\delta_{i}(z)$.

For $z_{0}=0$, we have

$$
\delta_{r}\left(z_{0}\right)=K K_{i}>0
$$

for $z_{j} \neq z_{0}$, where $j=1,2,3, \cdots$, we get

$$
\begin{gathered}
\delta_{r}\left(z_{j}\right)=K \frac{z_{j}^{2}}{L^{2}}\left[-K_{d}+m\left(z_{j}\right) K_{i}+b\left(z_{j}\right)\right]= \\
K \frac{z_{j}^{2}}{L^{2}}\left[-K_{d}+m_{j} K_{i}+b_{j}\right]
\end{gathered}
$$

where

$$
\left\{\begin{array}{l}
m\left(z_{j}\right)=m_{j} \\
b\left(z_{j}\right)=b_{j}
\end{array}\right.
$$

Interlacing the roots of $\delta_{r}(z)$ and $\delta_{i}(z)$ is equivalent to $\delta_{r}\left(z_{0}\right)>0\left(\right.$ since $\left.K_{i}>0\right), \delta_{r}\left(z_{1}\right)<0, \delta_{r}\left(z_{2}\right)>0, \cdots$.

We can use the interlacing property and the fact that $\delta_{i}(z)$ has only real roots to reach that $\delta_{r}(z)$ possesses real roots as well. From the previous equations, we get the fol- lowing inequalities

$$
\left\{\begin{array} { c } 
{ \delta _ { r } ( z _ { 0 } ) > 0 } \\
{ \delta _ { r } ( z _ { 1 } ) < 0 } \\
{ \delta _ { r } ( z _ { 2 } ) > 0 } \\
{ \delta _ { r } ( z _ { 3 } ) < 0 } \\
{ \delta _ { r } ( z _ { 4 } ) > 0 } \\
{ \vdots }
\end{array} \Rightarrow \left\{\begin{array}{c}
K_{i}>0 \\
K_{d}>m_{1} K_{i}+b_{1} \\
K_{d}<m_{2} K_{i}+b_{2} \\
K_{d}>m_{3} K_{i}+b_{3} \\
K_{d}<m_{4} K_{i}+b_{4} \\
\vdots
\end{array}\right.\right.
$$

Thus, intersecting all these regions in the $\left(K_{i}, K_{d}\right)$ space, we get the set of $\left(K_{i}, K_{d}\right)$ values for which the roots of $\delta_{r}(z)$ and $\delta_{i}(z)$ interlace for a fixed value of $K_{p}$. We notice that all these regions are half planes with their boundaries being lines with positive slopes $m_{j}$.

Example 2. Consider the plant given by (5) with the following parameters $K=2, a_{1}=5, a_{0}=-0.5$ and $L=0.5$.

$$
G(s)=\frac{2 \mathrm{e}^{-0.5 s}}{-0.5+5 s+s^{2}} .
$$

The imaginary part $\delta_{i}(z)$ has only simple real roots if and only if $0.25<K_{p}<7.85$. We set the controller parameter $K_{p}$ to 1.5 , which is inside the previous range. For this $K_{p}$, $\delta_{i}(z)$ takes the form:

$$
\begin{aligned}
\delta_{i}(z)= & \frac{z}{0.5}\left(2 K_{p}+\cos (z)\left(-0.5-\frac{z^{2}}{0.25}\right)-5 \frac{z}{0.5} \sin (z)\right)= \\
& 2 z\left[3+\cos (z)\left(-0.5-4 z^{2}\right)-10 z \sin (z)\right] .
\end{aligned}
$$

We next compute some of the positive real roots of this equation and arrange them in increasing order of magnitude:

$$
\begin{aligned}
& z_{0}=0, z_{1}=0.4375, z_{2}=2.292, z_{3}=5.185, z_{4}=8.141 \\
& z_{5}=11.22, z_{6}=14.31
\end{aligned}
$$

Using (29), we calculate the parameters $m_{j}$ and $b_{j}$ for $j=$ $1, \cdots, 6$ :

$$
\left\{\begin{array} { l } 
{ m _ { 1 } = 1 . 3 0 6 1 } \\
{ m _ { 2 } = 0 . 0 4 7 6 } \\
{ m _ { 3 } = 0 . 0 0 9 3 } \\
{ m _ { 4 } = 0 . 0 0 3 8 } \\
{ m _ { 5 } = 0 . 0 0 2 0 } \\
{ m _ { 6 } = 0 . 0 0 1 2 }
\end{array} \text { and } \quad \left\{\begin{array}{l}
b_{1}=-1.9581 \\
b_{2}=3.4130 \\
b_{3}=-5.7761 \\
b_{4}=8.5304 \\
b_{5}=-11.5059 \\
b_{6}=14.5353 .
\end{array}\right.\right.
$$

Interlacing of the roots of the real and the imaginary part occurs for $K_{p}=1.5$, if and only if the following inequalities are satisfied:

$$
\left\{\begin{array}{l}
K_{i}>0 \\
K_{d}>1.3061 K_{i}-1.9581 \\
K_{d}<0.0476 K_{i}+3.413 \\
K_{d}>0.0093 K_{i}-5.7761 \\
K_{d}<0.0038 K_{i}+8.5304 \\
K_{d}>0.002 K_{i}-11.5059 \\
K_{d}<0.0012 K_{i}+14.5353
\end{array}\right.
$$

The boundaries of these regions are illustrated in Fig. 8. 


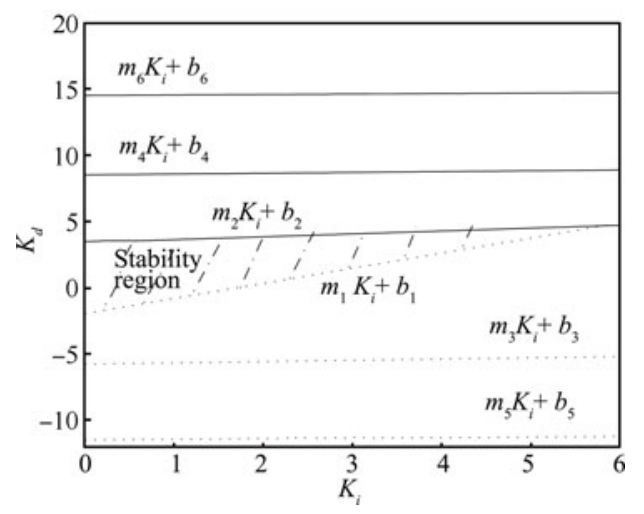

Fig. 8 Region boundaries of Example $2\left(K_{p}=1.5\right)$

Example 3. Consider the plant given by (5) with the following parameters $K=1, a_{1}=-0.1, a_{0}=0.5$ and $L=1$.

$$
G(s)=\frac{\mathrm{e}^{-s}}{0.5-0.1 s+s^{2}}
$$

The imaginary part $\delta_{i}(z)$ has only simple real roots if and only if $-0.5<K_{p}<0.2314$. We set the controller parameter $K_{p}$ to 0.1 , which is inside the previous range. For this $K_{p}, \delta_{i}(z)$ takes the form

$$
\delta_{i}(z)=z\left(0.1+\cos (z)\left(0.5-z^{2}\right)+0.1 z \sin (z)\right)=0 .
$$

We next compute some of the positive real roots of this equation and arrange them in increasing order of magnitude:

$$
\begin{aligned}
& z_{0}=0, z_{1}=0.8711, z_{2}=1.409, z_{3}=4.695, z_{4}=7.839 \\
& z_{5}=10.99, z_{6}=14.13
\end{aligned}
$$

Using (29), we calculate the parameters $m_{j}$ and $b_{j}$ for $j=$ $1, \cdots, 6$ :

$$
\left\{\begin{array} { l } 
{ m _ { 1 } = 1 . 3 1 7 8 } \\
{ m _ { 2 } = 0 . 5 0 3 7 } \\
{ m _ { 3 } = 0 . 0 4 5 4 } \\
{ m _ { 4 } = 0 . 0 1 6 3 } \\
{ m _ { 5 } = 0 . 0 0 8 3 } \\
{ m _ { 6 } = 0 . 0 0 5 }
\end{array} \text { and } \left\{\begin{array}{l}
b_{1}=0.2917 \\
b_{2}=1.0565 \\
b_{3}=-4.5895 \\
b_{4}=7.7758 \\
b_{5}=-10.9449 \\
b_{6}=14.095 .
\end{array}\right.\right.
$$

Interlacing the roots of the real and the imaginary part occurs for $K_{p}=0.1$, if and only if the following inequalities are satisfied:

$$
\left\{\begin{array}{l}
K_{i}>0 \\
K_{d}>1.3178 K_{i}+0.2917 \\
K_{d}<0.5037 K_{i}+1.0565 \\
K_{d}>0.0454 K_{i}-4.5895 \\
K_{d}<0.0163 K_{i}+7.7758 \\
K_{d}>0.0083 K_{i}-10.9449 \\
K_{d}<0.005 K_{i}+14.095
\end{array}\right.
$$

The boundaries of these regions are illustrated in Fig. 9.

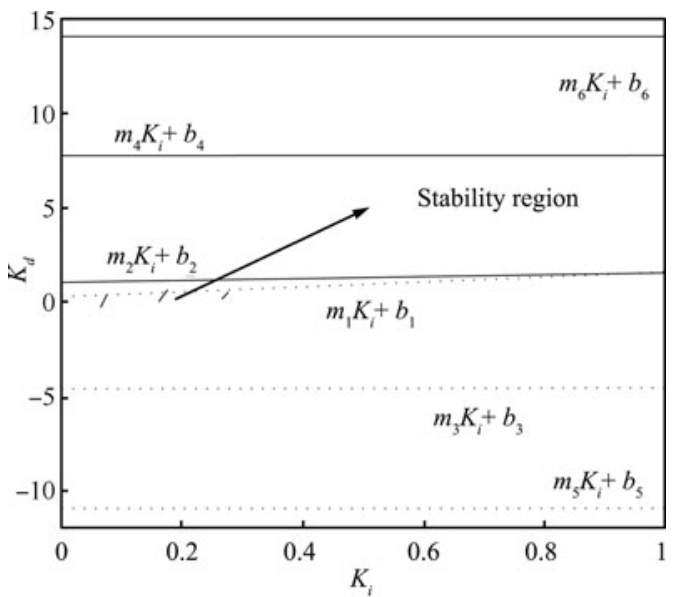

Fig. 9 Region boundaries of Example $3\left(K_{p}=0.1\right)$

An enlargement of Fig. 9 is shown in Fig. 10.

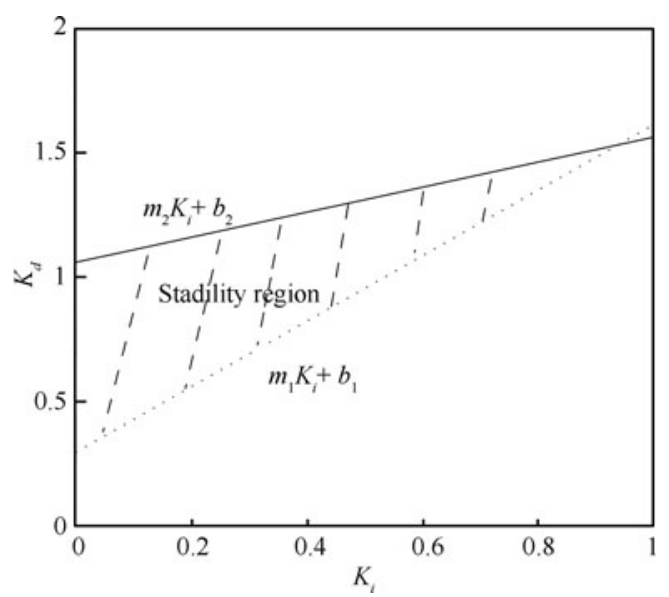

Fig. 10 Stability region of Example $3\left(K_{p}=0.1\right)$

Example 4. Consider the plant given by (5) with the following parameters $K=1, a_{1}=-0.1, a_{0}=-0.5$ and $L=1$.

$$
G(s)=\frac{\mathrm{e}^{-s}}{-0.5-0.1 s+s^{2}}
$$

The imaginary part $\delta_{i}(z)$ has only simple real roots if and only if $0.5<K_{p}<0.7442$. We set the controller parameter $K_{p}$ to 0.6 , which is inside the previous range. For this $K_{p}$, $\delta_{i}(z)$ takes the form

$$
z\left(-0.1+\cos (z)\left(-0.5-z^{2}\right)+0.1 z \sin (z)\right)=0 .
$$

We next compute some of the positive real roots of this equation and arrange them in increasing order of magnitude:

$z_{0}=0, z_{1}=0.4188, z_{2}=1.1916, z_{3}=4.718, z_{4}=7.8316$, $z_{5}=10.9915, z_{6}=14.1271$.

Using (29), we calculate the parameters $m_{j}$ and $b_{j}$ for $j=$ 
$1, \cdots, 6$ :

$$
\left\{\begin{array} { l } 
{ m _ { 1 } = 5 . 7 0 1 5 } \\
{ m _ { 2 } = 0 . 7 0 4 3 } \\
{ m _ { 3 } = 0 . 0 4 4 9 } \\
{ m _ { 4 } = 0 . 0 1 6 3 } \\
{ m _ { 5 } = 0 . 0 0 8 3 } \\
{ m _ { 6 } = 0 . 0 0 5 }
\end{array} \text { and } \left\{\begin{array}{l}
b_{1}=0.7472 \\
b_{2}=1.5338 \\
b_{3}=-4.8233 \\
b_{4}=7.8957 \\
b_{5}=-11.0373 \\
b_{6}=14.1628
\end{array}\right.\right.
$$

Interlacing the roots of the real and the imaginary part occurs for $K_{p}=0.6$, if and only if the following inequalities are satisfied:

$$
\left\{\begin{array}{l}
K_{i}>0 \\
K_{d}>5.7015 K_{i}+0.7472 \\
K_{d}<0.7043 K_{i}+1.5338 \\
K_{d}>0.0449 K_{i}-4.8233 \\
K_{d}<0.0163 K_{i}+7.8957 \\
K_{d}>0.0083 K_{i}-11.0373 \\
K_{d}<0.005 K_{i}+14.1628 .
\end{array}\right.
$$

The boundaries of these regions are illustrated in Fig. 11.

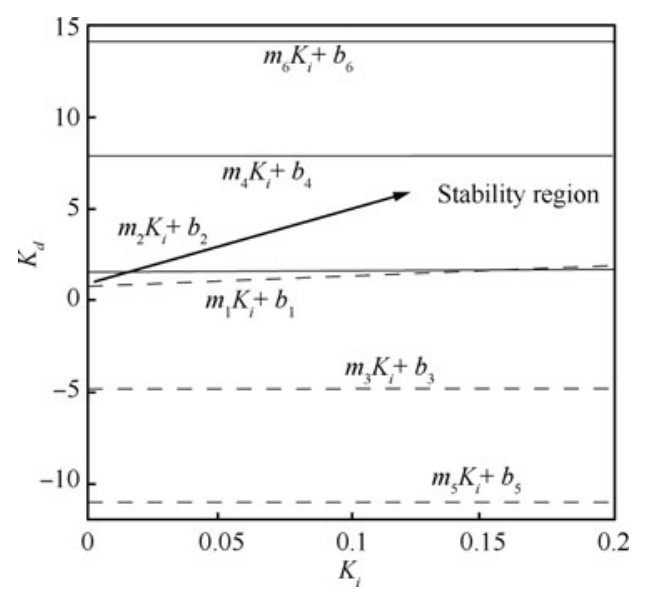

Fig. 11 Region boundaries of Example $4\left(K_{p}=0.6\right)$

An enlargement of Fig. 11 is shown in Fig. 12.

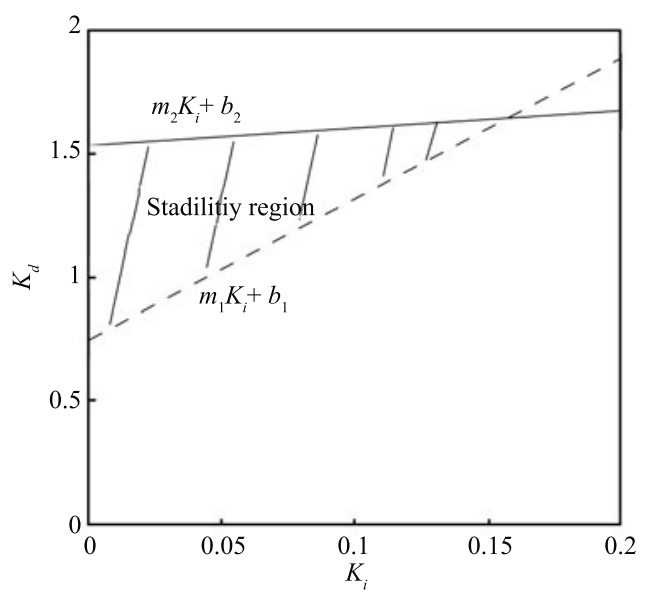

Fig. 12 Stability region of Example $4\left(K_{p}=0.6\right)$
The stability region is defined by only two boundaries

$$
\left\{\begin{array}{l}
K_{d}=m_{1} K_{i}+b_{1} \\
\text { and } \\
K_{d}=m_{2} K_{i}+b_{2}
\end{array}\right.
$$

because we have the following inequalities:

$$
\left\{\begin{array}{l}
b_{j}<b_{j+2}, \quad \text { for } j=2,4,6, \cdots \\
b_{j}>b_{j+2}, \quad \text { for } j=1,3,5,7, \cdots \\
m_{j}<m_{j+2}, \text { for } j \geqslant 1 .
\end{array}\right.
$$

As pointed out in Examples 2, 3 and 4, the inequalities given by (30) represent half planes in the space of $K_{i}$ and $K_{d}$. Their boundaries are given by lines with

$$
K_{d}=m_{j} K_{i}+b_{j} \text { for } j=1,2,3, \cdots \text {. }
$$

We now state an important technical lemma that allows us to develop an algorithm for solving the PID stabilization problem. This lemma shows the behavior of the parameter $b_{j}, j=1,2,3, \cdots$, for different values of the parameter $K_{p}$ inside the range proposed by Theorem 4 .

Lemma 1. If $-\frac{a_{0}}{K}<K_{p}<\frac{1}{K}\left(a_{1} \frac{\alpha}{L} \sin (\alpha)-\cos (\alpha)\left(a_{0}-\right.\right.$ $\left.\frac{\alpha^{2}}{L^{2}}\right)$ ), where $\alpha$ is the solution of the equation $\tan (\alpha)=$ $\frac{\alpha\left(2+a_{1} L\right)}{\left(\alpha^{2}-a_{1} L-a_{0} L^{2}\right)}$ in the interval $[0, \pi]$, then:

$$
\left\{\begin{array}{l}
1-b_{j}<b_{j+2}, \quad \text { for } j=2,4,6, \cdots \\
2-b_{j}>b_{j+2}, \text { for } j=1,3,5,7, \cdots \\
3-m_{j}>m_{j+2}, \text { for } j \geqslant 1 \text { and } \lim _{j \rightarrow \infty} m_{j}=0 \\
4-b_{1}>-\frac{a_{1}}{K} .
\end{array}\right.
$$

Proof. From equations (26) we have $m\left(z_{j}\right)=\frac{L^{2}}{z_{j}^{2}}, 0<$ $z_{j}<z_{j+2} \Rightarrow \frac{L^{2}}{z_{j}^{2}}>\frac{L^{2}}{z_{j+2}^{2}}$ for $j \geqslant 1$ then $m\left(z_{j}\right)>m\left(z_{j+2}\right)$ and $\lim _{j \rightarrow \infty} m\left(z_{j}\right)=0$.

Remark 1. As we can see from Fig. 1, the odd roots of $\delta_{i}(z)$, i.e., for $j=3,5,7, \cdots$, verify $z_{j} \in$ $\left[(2 j-3) \frac{\pi}{2},(j-1) \pi\right]$ and are getting closer to $(2 j-3) \frac{\pi}{2}$ as $j$ increases, so we have $\lim _{j \rightarrow \infty} \cos \left(z_{j}\right)=0$ and $\lim _{j \rightarrow \infty} \sin \left(z_{j}\right)=-1$.

Moreover, since cosine function and sine function are monotonically increasing between $(2 j-3) \frac{\pi}{2}$ and $(j-1) \pi$, we have

$$
\begin{aligned}
& \left\{\begin{array}{l}
\cos \left(z_{3}\right)>\cos \left(z_{5}\right)>\cos \left(z_{7}\right)>\cdots \\
\sin \left(z_{3}\right)>\sin \left(z_{5}\right)>\sin \left(z_{7}\right)>\cdots
\end{array} \Rightarrow\right. \\
& \left\{\begin{array}{l}
\cos \left(z_{j}\right)>\cos \left(z_{j+2}\right)>0 \\
\sin \left(z_{j+2}\right)<\sin \left(z_{j}\right)<0
\end{array}\right.
\end{aligned}
$$

Remark 2. As we can see from Fig. 1, the even roots of $\delta_{i}(z)$, i.e., for $j=2,4,6, \cdots$, verify $z_{j} \in$ $\left[(2 j-3) \frac{\pi}{2},(j-1) \pi\right]$ and are getting closer to $(2 j-3) \frac{\pi}{2}$ as $j$ increase, so we have $\lim _{j \rightarrow \infty} \cos \left(z_{j}\right)=0$ and $\lim _{j \rightarrow \infty} \sin \left(z_{j}\right)=1$.

Moreover, since cosine function and sine function are monotonically decreasing between $(2 j-3) \frac{\pi}{2}$ and $(j-1) \pi$, 
we have

$$
\begin{aligned}
& \left\{\begin{array}{l}
\cos \left(z_{2}\right)<\cos \left(z_{4}\right)<\cos \left(z_{6}\right)<\cdots \\
\sin \left(z_{2}\right)<\sin \left(z_{4}\right)<\sin \left(z_{6}\right)<\cdots
\end{array} \Rightarrow\right. \\
& \left\{\begin{array}{l}
\cos \left(z_{j}\right)<\cos \left(z_{j+2}\right)<0 \\
0<\sin \left(z_{j}\right)<\sin \left(z_{j+2}\right)
\end{array}\right.
\end{aligned}
$$

The change of $b\left(z_{j}\right)$ can be found with graphical approach. Given a stabilizing $K_{p}$ value inside the admissible range by using Theorem 4 ; the curve of $\delta_{i}(z)$ is plotted to obtain its roots denoted by $z_{j}=1,2,3, \cdots$, the curves of $-a_{1} \frac{z}{L} \cos (z)$ and $\sin (z)\left(\frac{z^{2}}{L^{2}}-a_{0}\right)$ are plotted as well in order to understand the behavior of $b\left(z_{j}\right)$ (Fig. 13).

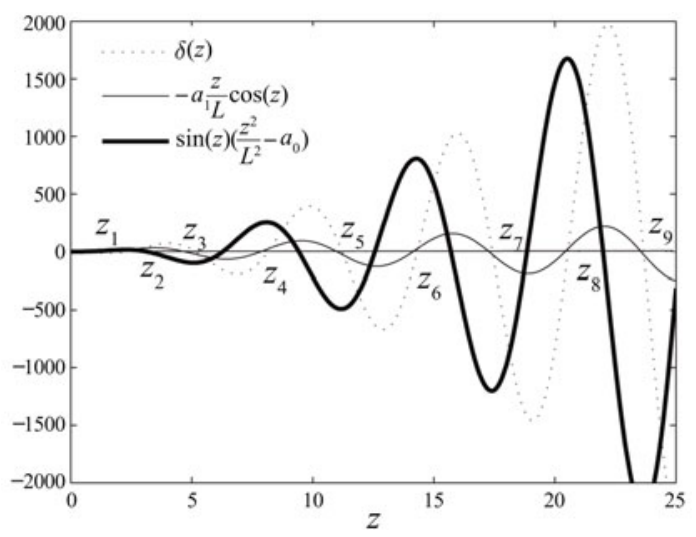

Fig. 13 Plots of $\delta_{i}(z)$ (dotted line), $\sin (z)\left(\frac{z^{2}}{L^{2}}-a_{0}\right)$ (thick solid line) and $-a_{1} \frac{z_{j}}{L} \cos \left(z_{j}\right)$ (thin solid line)

As far as the odd roots of $\delta_{i}(z)$ are concerned, the corresponding $\sin (z)\left(\frac{z^{2}}{L^{2}}-a_{0}\right)$ is decreasing by large magnitude $\sin \left(z_{j}\right)\left(\frac{z_{j}^{2}}{L^{2}}-a_{0}\right) \rightarrow-\infty$ as $j \rightarrow+\infty$, and for the even ones, the corresponding $\sin (z)\left(\frac{z^{2}}{L^{2}}-a_{0}\right)$ is increasing by large magnitude $\sin \left(z_{j}\right)\left(\frac{z_{j}^{2}}{L^{2}}-a_{0}\right) \rightarrow+\infty$ as $j \rightarrow+\infty$. However, compared to the change of $\sin \left(z_{j}\right)\left(\frac{z_{j}^{2}}{L^{2}}-a_{0}\right)$, the difference between the values of $-a_{1} \frac{z_{j+2}}{L} \cos \left(z_{j+2}\right)$ and $-a_{1} \frac{z_{j}}{L} \cos \left(z_{j}\right)$ is much smaller than both odd and even $j$. Thus, the $b\left(z_{j}\right)$ has the similar change rules as $\sin \left(z_{j}\right)\left(\frac{z_{j}^{2}}{L^{2}}-\right.$ $\left.a_{0}\right)$.

We can affirm that: $j$.

1) $b_{j}>b_{j+2}$ and $b_{j} \rightarrow-\infty$ as $j \rightarrow+\infty$ for odd values of

2) $b_{j}<b_{j+2}$ and $b_{j} \rightarrow+\infty$ as $j \rightarrow+\infty$ for even values of $j$.

Remark 3. Let us prove that $b\left(z_{j}\right)>-\frac{a_{1}}{K}$ for $j=$ $1,3,5,7, \cdots$, we have

$$
\left\{\begin{array} { c } 
{ z _ { 1 } \in [ 0 , \frac { \pi } { 2 } ] } \\
{ z _ { 3 } \in [ \frac { 3 \pi } { 2 } , 2 \pi ] } \\
{ z _ { 5 } \in [ \frac { 7 \pi } { 2 } , 4 \pi ] } \\
{ \vdots }
\end{array} \quad \text { and } \left\{\begin{array}{c}
z_{2} \in\left[\frac{\pi}{2}, \pi\right] \\
z_{4} \in\left[\frac{5 \pi}{2}, 3 \pi\right] \\
z_{6} \in\left[\frac{9 \pi}{2}, 5 \pi\right] \\
\vdots
\end{array}\right.\right.
$$

i.e., $z_{j}$ verifies

$$
\left\{\begin{array}{l}
z_{1} \in\left[0, \frac{\pi}{2}\right] \\
\text { and } \\
z_{j} \in\left[(2 j-3) \frac{\pi}{2},(j-1) \pi\right], \text { for } j \geqslant 2 .
\end{array}\right.
$$

For $z_{1}$, we have $\cos \left(z_{1}\right)>0$ and $\sin \left(z_{1}\right)>0$.

Case 1. $a_{0}<0$ and $a_{1}>0$.

We suppose

$$
\begin{aligned}
& b\left(z_{1}\right)<-\frac{a_{1}}{K} \Rightarrow \\
& \frac{L}{K z}\left[-a_{1} \frac{z_{1}}{L} \cos \left(z_{1}\right)+\sin \left(z_{1}\right)\left(\frac{z_{1}^{2}}{L^{2}}-a_{0}\right)\right]<-\frac{a_{1}}{K} \Rightarrow \\
& -a_{1} \frac{z_{1}}{L} \cos (z)+\sin \left(z_{1}\right)\left(\frac{z_{1}^{2}}{L^{2}}-a_{0}\right)<-a_{1} \frac{z_{1}}{L} \Rightarrow \\
& a_{1} \frac{z_{1}}{L}\left(1-\cos \left(z_{1}\right)\right)+\sin \left(z_{1}\right)\left(\frac{z_{1}^{2}}{L^{2}}-a_{0}\right)<0
\end{aligned}
$$

which is wrong because we have

$$
\left\{\begin{array}{l}
a_{1} \frac{z_{1}}{L}\left(1-\cos \left(z_{1}\right)\right)>0 \\
\text { and } \\
\sin \left(z_{1}\right)\left(\frac{z_{1}^{2}}{L^{2}}-a_{0}\right)>0
\end{array}\right.
$$

so $b\left(z_{1}\right)>-\frac{a_{1}}{K}$.

Case 2. $a_{0}>0$ and $a_{1}<0$.

For $z_{1}$, we have $\cos \left(z_{1}\right)>0, \sin \left(z_{1}\right)>0$, and in this case, we notice that $z_{1}<L \sqrt{a_{0}}$.

$$
\begin{aligned}
& {\left[-a_{1} \frac{z_{1}}{L} \cos \left(z_{1}\right)+\sin \left(z_{1}\right)\left(\frac{z_{1}^{2}}{L^{2}}-a_{0}\right)\right]>} \\
& \quad-a_{1} \frac{z_{1}}{L} \cos \left(z_{1}\right)>-a_{1} \frac{z_{1}}{L} \Rightarrow \\
& \quad \frac{L}{K z_{1}}\left[-a_{1} \frac{z_{1}}{L} \cos \left(z_{1}\right)+\sin \left(z_{1}\right)\left(\frac{z_{1}^{2}}{L^{2}}-a_{0}\right)\right]>-\frac{a_{1}}{K} \Rightarrow \\
& b\left(z_{1}\right)=b_{1}>-\frac{a_{1}}{K} .
\end{aligned}
$$

Case 3. $a_{0}<0$ and $a_{1}<0$.

$$
\begin{aligned}
& {\left[-a_{1} \frac{z_{1}}{L} \cos \left(z_{1}\right)+\sin \left(z_{1}\right)\left(\frac{z_{1}^{2}}{L^{2}}-a_{0}\right)\right]>} \\
& \quad-a_{1} \frac{z_{1}}{L} \cos \left(z_{1}\right)>-a_{1} \frac{z_{1}}{L} \Rightarrow \\
& \quad \frac{L}{K z_{1}}\left[-a_{1} \frac{z_{1}}{L} \cos \left(z_{1}\right)+\sin \left(z_{1}\right)\left(\frac{z_{1}^{2}}{L^{2}}-a_{0}\right)\right]>-\frac{a_{1}}{K} \Rightarrow \\
& b\left(z_{1}\right)=b_{1}>-\frac{a_{1}}{K}
\end{aligned}
$$

We conclude that

$$
b\left(z_{1}\right)>-\frac{a_{1}}{K} .
$$

By using (32) and (33), the stability region defined by (30) can be reduced to the following boundaries:

$$
\left\{\begin{array}{l}
K_{d}>m_{1} K_{i}+b_{1} \\
K_{d}<m_{2} K_{i}+b_{2} \\
K_{d}>-\frac{a_{1}}{K}
\end{array}\right.
$$


In view of Theorem 4, we propose an algorithm to determine the set of all stabilizing PID parameters for an unstable second order delay system.

Algorithm 2. For determining PID parameters for unstable second order delay system:

1) Choose $K_{p}$ in the interval suggested by Theorem 4;

2) Find the roots $z_{j}$ of $\delta_{i}(z)$;

3) Compute the parameters $m_{j}$ and $b_{j}, j=1,2$, associated with the $z_{j}$ previously found;

4) Determine the stability region in the plane $\left(K_{i}, K_{d}\right)$ using Fig. 7 (Theorem 4);

5) Go to step 1.

Example 5. Finally, let us use the results of this section to determine the entire set of PID parameters to stabilize the unstable delay system which is presented in Example 2. The range of $K_{p}$ values specified by Theorem 4 is given by

$$
-0.5<K_{p}<0.2314
$$

By sweeping over this range and using the algorithm presented earlier, we obtain the stabilizing set of $\left(K_{p}, K_{i}, K_{d}\right)$ values sketched in Fig. 14.

Example 6. Consider an unstable process as given by Huang and Chen ${ }^{[32]}$

$G(s)=\frac{\mathrm{e}^{-0.939 s}}{(5 s-1)(2.07 s+1)}=\frac{0.0966}{\mathrm{e}^{-0.939 s}} s^{2}+2.93 s-0.0966$.

We present a comparison of different methods for design of PID controllers for unstable second order delay system.

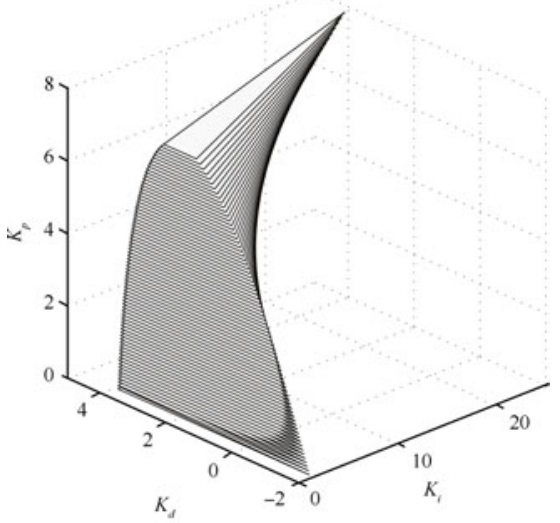

Fig. 14 The stabilizing region of $\left(K_{p}, K_{i}, K_{d}\right)$ values for the PID controller in Example 2

By using our approach, the entire set of PID parameters stabilizing $G(s)$ is obtained by the following figure.

We observe that the PID tuning values of some existing methods in Table 1 are included in the stability region given by Fig. 15 .

Table 1 Performance comparison

\begin{tabular}{cccc}
\hline Method & $K_{p}$ & $K_{i}$ & $K_{d}$ \\
\hline Huang and Chen $^{[32]}$ & 6.186 & 0.8628 & 9.1058 \\
Huang and Lin $^{[33]}$ & 3.954 & 0.7975 & 8.2006 \\
Poulin and Pomerleau $^{[12]}$ & 3.050 & 0.4036 & 6.3135 \\
Lee et al. ${ }^{[34]}$ & 7.144 & 1.0688 & 11.8233 \\
\hline
\end{tabular}

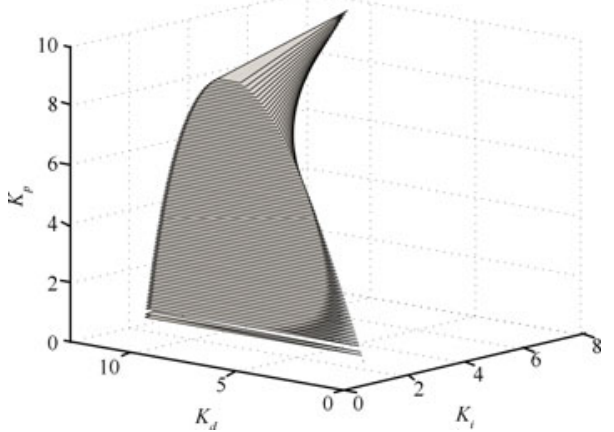

Fig. 15 The stabilizing region of $\left(K_{p}, K_{i}, K_{d}\right)$ values for the PID controller

1) Method of Huang and Chen ${ }^{[32]}: K_{p}=6.186, K_{i}=$ $0.8628, K_{d}=9.1058$.

These PID tuning parameters are included in the stability region in the $\left(K_{i}, K_{d}\right)$ plane for a fixed $K_{p}=6.186$ (see Fig. 16).

2) Method of Huang and $\operatorname{Lin}^{[33]}: K_{p}=3.954, K_{i}=$ $0.7975, K_{d}=8.2006$.

These PID tuning parameters are included in the stability region in the $\left(K_{i}, K_{d}\right)$ plane for a fixed $K_{p}=3.954$ (see Fig. 17).

3) Method of Poulin and Pomerleau ${ }^{[12]}: \quad K_{p}=$ $3.050, K_{i}=0.4036, K_{d}=6.3135$.

These PID tuning parameters are included in the stability region in the $\left(K_{i}, K_{d}\right)$ plane for a fixed $K_{p}=3.050$ (see Fig. 18)

4) Method of Lee et al. ${ }^{[34]}: \quad K_{p}=7.144, K_{i}=$ $1.0688, K_{d}=11.8233$.

These PID tuning parameters are included in the stability region in the $\left(K_{i}, K_{d}\right)$ plane for a fixed $K_{p}=7.144$ (see Fig. 19).

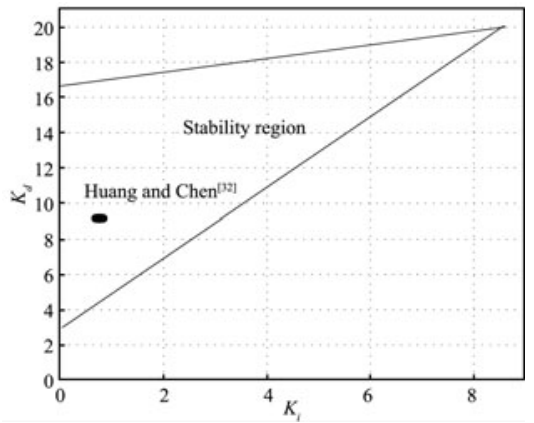

Fig. 16 Stability region in $\left(K_{i}, K_{d}\right)$ plane for a $K_{p}=6.186$

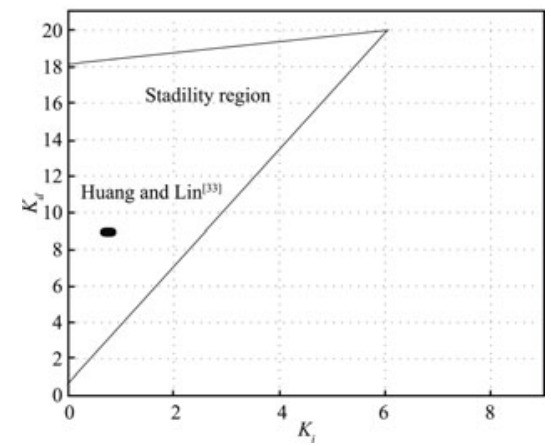

Fig. 17 Stability region in $\left(K_{i}, K_{d}\right)$ plane for a $K_{p}=3.954$ 


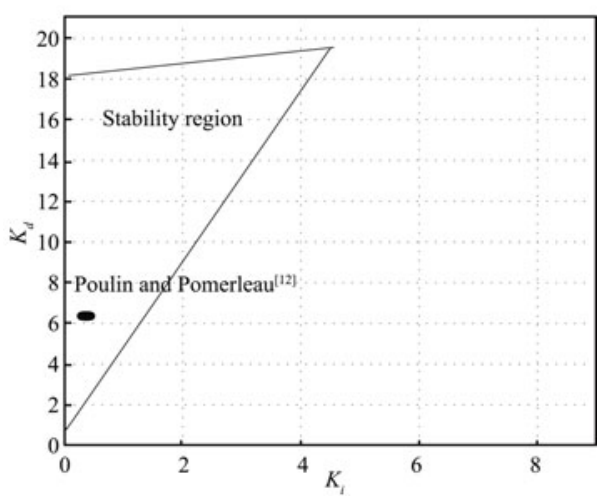

Fig. 18 Stability region in $\left(K_{i}, K_{d}\right)$ plane for a $K_{p}=3.050$

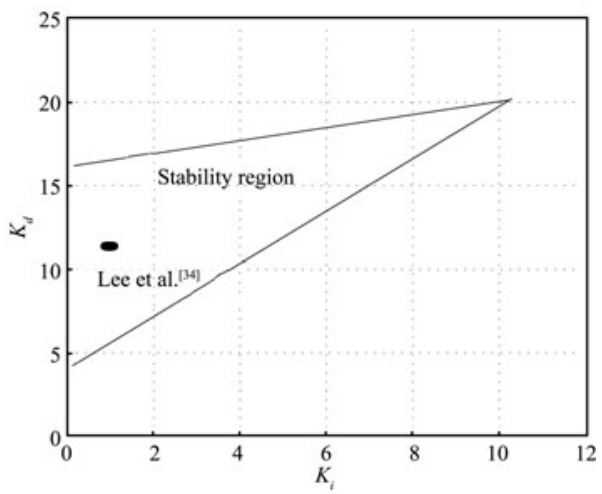

Fig. 19 Stability region in $\left(K_{i}, K_{d}\right)$ plane for a $K_{p}=7.144$

\section{Conclusion}

In this work, we proposed an extension of HermiteBiehler theorem to compute the region of stability for unstable second order delay system controlled by PI and PID controllers. Firstly, the procedure is based on determining the range of proportional gain value $K_{p}$ for which a solution to PID stabilization problem exists. Then, it is shown that for a fixed $K_{p}$ inside this range, the stabilizing integral $K_{i}$ and derivative gain $K_{d}$ values lie inside a region with known shape and boundaries.

\section{References}

[1] S. I. Niculescu. Delay Effects on Stability, London: Springer, 2001

[2] Q. C. Zhong. Robust Control of Time Delay System, London: Springer, 2006

[3] M. Chidambaram. Control of unstable systems: A review. Journal of Energy Heat and Mass Transfer, vol. 19, pp. 49$56,1997$.

[4] W. L. Bialkowski. Control of the pulp and paper making process. The Control Handbook, W. S. Levine, Ed., Boca Raton, Florida, USA: CRC/IEEE Press, pp.12191242, 1996.

[5] K. J. AAström, T. Hägglund. PID Controllers: Theory, Design, and Tuning, 2nd ed., Research Triangle Park, NC: Instrument Society of America, 1995.
[6] H. Takatsu, T. Itoh, M. Araki. Future needs for the control theory in industries-report and topics of the control technology survey in Japanese industry. Journal of Process Control, vol. 8, no. 5-6, pp.369-374, 1998.

[7] A. M. De Paor, M. O'Malley. Controllers of Ziegler-Nichols type for unstable process with time delay. International Journal of Control, vol. 49, no. 4, pp. 1273-1284, 1989.

[8] S. B. Jr. Quinn, C. K. Sanathanan. Controller design for integrating and runaway processes involving time delay. AIChE Journal, vol. 35, no. 6, pp. 923-930, 1989.

[9] Z. Shafiei, A. T. Shenton. Tuning of PID-type controllers for stable and unstable systems with time delay. Automatica, vol. 30, no. 10, pp. 1609-1615, 1994.

[10] R. Padma Sree, M. N. Sirinivas, M. Chidambaram. A simple method of tuning PID controllers for stable and unstable FOPTD systems. Computers and Chemical Engineering, vol. 28, no. 11, pp. 2201-2218, 2004.

[11] C. T. Huang, Y. S. Lin. Tuning PID controller for open-loop unstable processes with time delay. Chemical Engineering Communications, vol.133, no. 11, pp. 11-13, 1995.

[12] E. D. Poulin, A. Pomerleau. PID tuning for integrating and unstable processes. IEE Proceedings - Control Theory and Application, vol. 143, no. 5, pp. 429-434, 1996.

[13] C. Hwang, J. H. Hwang. Stabilisation of first-order plus dead-time unstable processes using PID controllers. IEE Proceedings - Control Theory and Application, vol.151, no. 1, pp. 89-94, 2004.

[14] C. Xiang, Q. G. Wang, X. Lu, L. A. Nguyen, T. H. Lee. Stabilization of second-order unstable delay processes by simple controllers. Journal of Process Control, vol. 17, no. 8, pp. 675-682, 2007.

[15] A. S. Rao, M. Chidambaram. Enhanced two-degreesoffreedom control strategy for second-order unstable processes with time delay. Industrial and Engineering Chemistry Research, vol. 45, no. 10, pp. 3604-3614, 2006.

[16] C. C. Chen, H. P. Huang, H. J. Liaw. Set-point weighted PID controller tuning for time-delayed unstable processes. Industrial and Engineering Chemistry Research, vol. 47, no. 18, pp. 6983-6990, 2008.

[17] M. Shamsuzzoha, J. Jeon, M. Le. Improved analytical PID controller design for the second order unstable process with time delay. Computer Aided Chemical Engineering, vol. 24, pp. 901-906, 2007.

[18] R. C. Panda. Synthesis of PID controller for unstable and integrating processes. Chemical Engineering Science, vol. 64 , no. 12 , pp. $2807-2816,2009$.

[19] C. L. Zhang, J. M. Li. Adaptive iterative learning control for nonlinear time-delay systems with periodic disturbances using FSE-neural network. International Journal of Automation and Computing, vol. 8, no. 4, pp. 403-410, 2011.

[20] W. S. Chen, R. H. Li. Observer-based adaptive iterative learning control for nonlinear systems with time-varying delays. International Journal of Automation and Computing, vol. 7 , no. 4, pp. 438-446, 2010.

[21] Q. W. Deng, Q. Wei, Z. X. Li. Analysis of absolute stability for time-delay teleoperation systems. International Journal of Automation and Computing, vol.4, no. 2, pp. 203-207, 2007. 
[22] V. Kumar, A. P. Mittal. Parallel fuzzy P + fuzzy I + fuzzy D controller: Design and performance evaluation. International Journal of Automation and Computing, vol. 7, no. 4, pp. 463-471, 2010

[23] G. J. Silva, A. Datta, S. P. Bhattacharyya. PI stabilization of first-order systems with time delay. Automatica, vol. 37, no. 12, pp. 2025-2031, 2001.

[24] G. J. Silva, A. Datta, S. P. Bhattacharyya. New results synthesis of PID controller. IEEE Transactions on Automatic Control, vol. 47, no. 2, pp. 241-252, 2002.

[25] G. J. Silva, A. Datta, S. P. Bhattacharyya. PID Controllers for Time Delay Systems, London: Springer, 2005.

[26] G. J. Silva, A. Datta, S. P. Bhattacharyya. Stabilization of time delay systems. In Proceedings of the American Control Conference, IEEE, Chicago, Illinois, USA, pp. 963-970, 2000.

[27] G. J. Silva, A. Datta, S. P. Bhattacharyya. Stabilization of first-order systems with time delay using the PID controller. In Proceedings of the American Control Conference, IEEE, Arlington, VA, USA, pp. 4650-4655, 2001.

[28] R. Farkh, K. Laabidi, M. Ksouri. PI control for second order delay system with tuning parameter optimization. International Journal of Electrical and Electronics Engineering, vol. 3, pp. 1-7, 2009

[29] R. Farkh, K. Laabidi, M. Ksouri. Computation of all stabilizing PID gain for second-order delay system. Mathematical Problem in Engineering, vol. 2009, Article ID 212053, 17 pages, 2009.

[30] R. Farkh, K. Laabidi, M. Ksouri. Robust stabilization for uncertain second order time-lag system. The Mediterranean Journal of Measurement and Control, vol. 5, no. 4, pp. 138$145,2009$.

[31] R. Farkh, K. Laabidi, M. Ksouri. Robust PI/PID controller for interval first order system with time delay. International Journal of Modelling Identification and Control, vol. 13, no. 1-2, pp. 67-77, 2011.

[32] H. P. Huang, C. C. Chen. Control-system synthesis for open-loop unstable process with time delay. IEE Proceedings - Control Theory and Application, vol. 144, no. 4, pp. 334-346, 1997.

[33] C. T. Huang, Y. S. Lin. Tuning PID controller for open-loop unstable processes with time delay. Chemical Engineering Communications, vol.133, no. 11, pp.11-30, 1995.
[34] Y. Lee, J. Lee, S. Park. PID controller tuning for integrating and unstable processes with time delay. Chemical Engineering Science, vol. 55, no. 17, pp. 3481-3493, 2000.

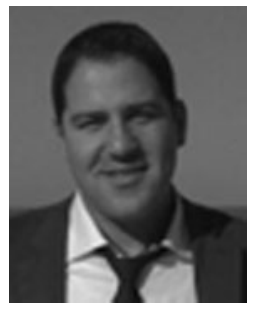

Rihem Farkh received his engineer degree in electrical engineering, his M. Sc. degree in automatic control and signal processing and his Ph. D. degree in electrical engineering from the National Engineering School of Tunis, Tunis El-Manar University, Tunisia in 2006, 2007 and 2011, respectively. He is currently an assistant professor at Sousse University, Tunisia.

His research interests include PID control, robust control and time-delay systems.

E-mail: rihem.farkh@enit.rnu.tn (Corresponding author)

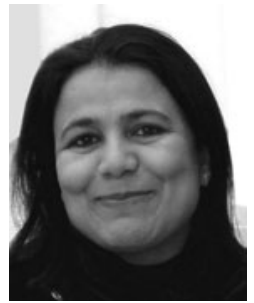

Kaouther Laabidi received her master degree from the Higher School of Sciences and Technologies of Tunis in 1995, her $\mathrm{Ph}$. D. degree and the post-doctoral degree allowing to supervise Ph. D. in electrical engineering from the National Engineering School of Tunis, Tunis El-Manar University in 2005 and 2011, respectively. She is currently an associate professor at Tunis University, Tunisia.

Her research interests include identification and control of complex systems.

E-mail: Kaouther.Laabidi@enit.rnu.tn

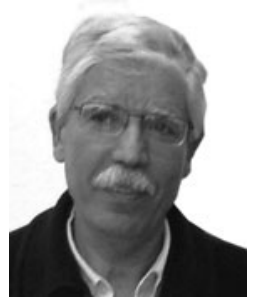

Mekki Ksouri received his M. A. degree in physics from the Faculty of Mathematical, Physical and Natural Sciences of Tunis in 1973, his M. Eng. degree from the High School of Electricity in Paris in 1973 his D. Sc. degree, and Ph. D. degree from the University of Paris VI, Paris, France, in 1975 and 1977, respectively. He is professor at the National Engineering School of Tunis (ENIT). He was principal of the National Institute of Applied Sciences and Technology (INSAT) from 1999 to 2005, principal and founder of the High School of Statistics and Information Analysis from 2001 to 2005, and the High Institute of Technologic Studies from 1996 to 1999, and principal of the High Normal School of Technological Education from 1978 to 1990 . He is the author or coauthor of many journal articles, book chapters, and communications in international conferences. He is also the author of 6 books.

His research interests include robust control, time delay system, predictive and adaptive control, Bond graph, multi-model.

E-mail: Mekki.Ksouri@enit.rnu.tn 